\title{
Plants used as Ethnoveterinary Medicines in Sikkim Himalayas
}

\author{
Kumar Avinash Bharati \& B.L. Sharma
}

\section{Research}

\begin{abstract}
Field work was conducted to document the ethnoveterinary medicine used by members of the indigenous community in Sikkim Himalayas, India, in order to treat ailments of their livestock. This research detailed the use of 37 medicinal plants to treat ailments in animals such as diarrhea, dysentery, digestive disorders, injury, wound, fever, maternity complications, skin disease, urinary problems, cough and cold, skeleto-muscular disorders, inflammation, scorpion sting, snake and insect bite, weakness, parasite, ulcer and bleeding. 12 medicinal plants being used in Sikkim Himalayas have not been documented in ethnoveterinary medicine elsewhere in the world. 15 plant species were found to contain previously unreported medicinal properties.
\end{abstract}

\section{Introduction}

Folk veterinary practices are seen as a holistic approach for livestock health care and management needs by the local indigenous population in Sikkim Himalayas. These practices have been transmitted orally from one generation to the next. In India, ancient literature such as the Vedas, and other written scriptures like Scand Puran (1000 BC), Devi Puran (2350 BC), Cherak and Shusruta (2500$600 \mathrm{BC}$ ) have long documented the treatment of animal disease by using medicinal plants (Dwivedi 2003, Kumar 2003, Shirlaw 1940, Swarup 2003). Modern research continues to contribute to this body of knowledge such as, Biswas (1956), Chopra et al. (1956), Kirtikar and Basu (1933) and Jain (1981). Research focusing on ethnoveternary medines also continues, with some interesting work having been done by Gaur (1992), Jain and Srivastava (1999), Kiruba et al. (2006), Pande et al. (2007), Srivastava et al. (2000).
Sikkim is a state in India, situated on the flanks of the eastern Himalayas between 27010' - 2805' N latitude and 88030 ' - 890 E longitude, bordered by China (Tibet) to the north-east, Bhutan to the south-east and Nepal to the west. Towards the south is the Indian state of West Bengal (Figure 1). Sikkim is geographically diverse, ranging in altitude from $244 \mathrm{~m}$ to $8598 \mathrm{~m}$. The climate ranges from tropical to high alpine. Owing to its range of altitude the vegetation of Sikkim may be classified into five categories: Low Hill Forests (tropical to subtropical type i.e. Terai forest, up to $900 \mathrm{~m}$ ), Middle Hill Forests (subtropical type, 750-1500 m), Upper Hill forests (Warm or wet temperate type 1500$2700 \mathrm{~m}$ ), Rhododendron-Conifer Zone (cold temperate or sub-alpine, 2700-3600 m), Alpine Scrub and Grasslands (3600-4300m and above) (Chaudhary 1951).

Sikkim possesses high plant diversity and is listed as one of the world's 25 biodiversity hotspots (Myers et al. 2000). Although it consists of only $0.2 \%$ of the total geographical area of India it has as many as 5,000 species of flowering plants and 362 species of ferns and ferns allies (Hara 1966, Mehra \& Bir 1964, Rai \& Sharma 1994, Singh \&

\section{Correspondence}

Kumar Avinash Bharati, Raw Materials Herbarium and Museum Delhi (RHMD), CSIR-National Institute of Science Communication and Information Resources, Dr. K.S. Krishnan Marg, New Delhi-110012, INDIA. kumaravinashbharati@rediffmail.com

B.L. Sharma, Department of Botany, N.R.E.C. College, Khurja-203131, INDIA.

Ethnobotany Research \& Applications 10:339-356 (2012)

Published: August 6, 2012

www.ethnobotanyjournal.org/vol10/i1547-3465-10-339.pdf 


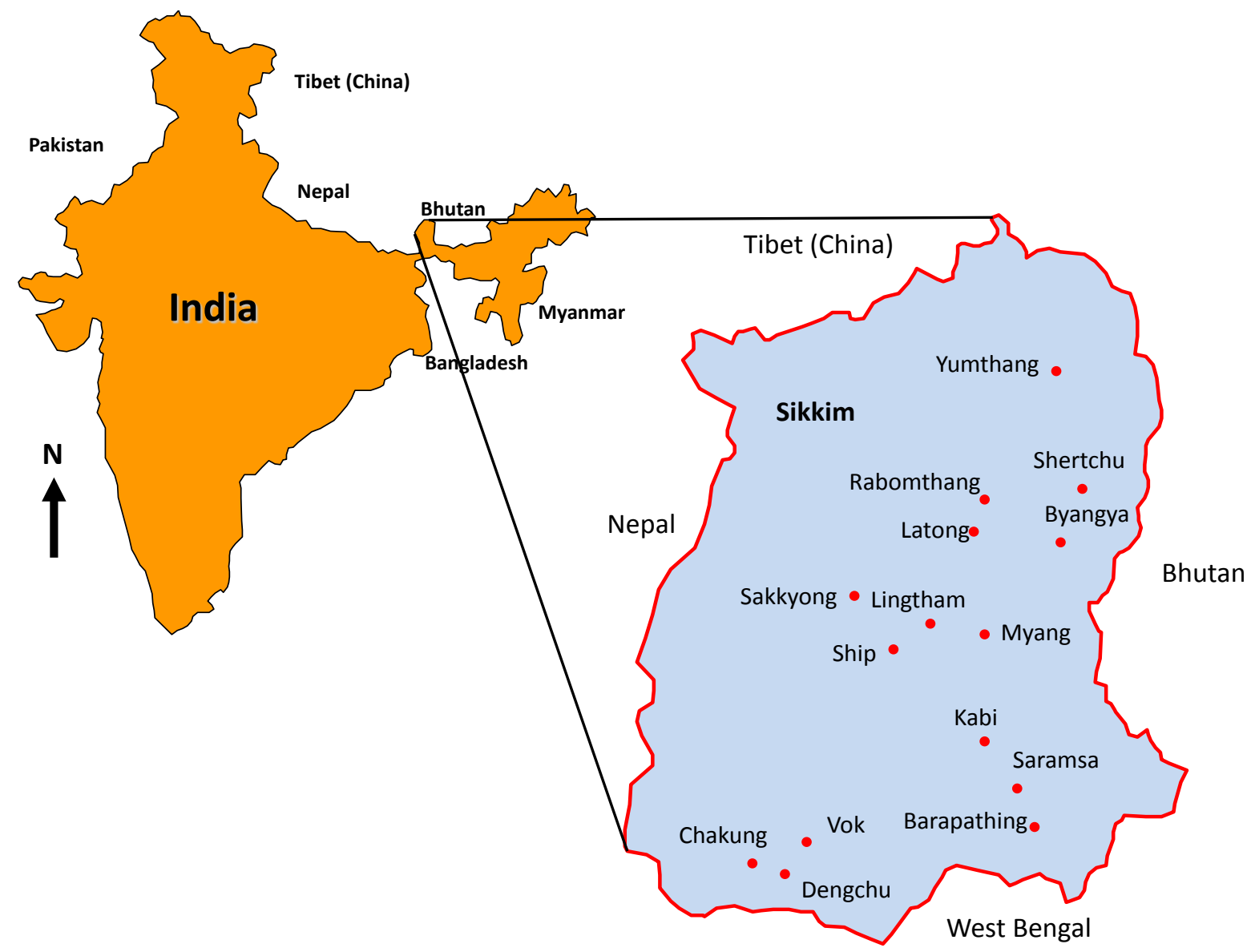

Figure 1. Sikkim, India, showing the villages surveyed for ethnoveterinary medicines.

Chauhan 1997). Sikkim's vegetation is divided into three altitudinal types, tropical (600-1500 m ASL), temperate (2700-3900 m ASL), and alpine (4000-5500 m ASL). Most of the areas of the state are very wet during June to September (Hajra \& Verma 1996). On average, the eastern and southern portions get a heavier rainfall (3040-3810 $\mathrm{mm})$ than the western portion of the state $(2030-2540$ $\mathrm{mm})$ (Chaudhary 1951).

The native population of Sikkim is comprised mainly of three groups, Bhutias, Lepchas and Nepalese (Rai \& Sharma 1994). The Lepchas are the earliest known inhabitants of the Sikkim. According to the 1891 census Lepchas accounted for $19 \%$ of the population. Recent census data shows they now only account for $8 \%$ (about 30,000) of the total population of Sikkim (Anonymous 2008). The Lepcha are mainly concentrated in the Dzongu reserve of the North district. The concept of health and illness among the Lepchas is guided by a belief in the supernatural. Before ethnoveternary treatments are begun, sacred hymns are chanted in front of the livestock by religious healers known as Lama (priest), Mun and Bongthing. These healers are considered by the Lepchas to be the mediums for communicating to the gods (rum) and devils or de- mons (Mungs). They also believe that the sacred chants ward off evil spirits and protect their livestock. Almost all Sikkimese, regardless of ethnic group, believe in the use of sacred chants to keep evil spirits at bay, and for successful treatments. They also use Dacho (prayer flags) for good luck and protection from demons (Burman 2003).

Bhutias make up about $14 \%$ of Sikkim's total population. They live primarily in some of the inner dry valleys of northern Sikkim. Their main sources of livelihood are animal husbandry and trade. Bhutias often reside in separate hamlets of villages. Although their culture shares many attributes with Tibetans, they have a distinct identity and language called Sikkimese. The religious doctors of Bhutias are known as pau (male), neyjum (female), and lama/priests, who treat patients with chants, tantras and herbs.

The eastern and southern parts of the state are larglely inhabited by the Nepalese, the majority ethnic group (about $70 \%$ of population) of the state. Nepalese communities are chiefly agriculturalists (Chaudhary 1951). A majority of the Nepalese community are Hindus with a few Buddhists. Nepali is the primary language of this Nepal- 


\section{Bharati \& Sharma - Plants used as Ethnoveterinary Medicines in Sikkim Himalayas}

ese community. The native doctors in this community are known as Jhankri.

Native peoples of Sikkim are highly dependent on animal husbandry, as it plays a major role in their livelihoods, especially at higher altitudes. A large number of livestock are still maintained using the traditional methods of the region (Balaraman \& Golay 1991). The major livestock of the state are cattle $(51 \%)$, goats $(29 \%)$, pigs $(9 \%)$, dogs $(6 \%)$, sheep $(3 \%)$, horses \& ponies $(1 \%)$, buffalos $(1 \%)$, yaks (1\%) and poultry (Anonymous 2006$)$.

The area of Sikkim remained largely isolated due to its geographical impasses until the middle of the $19^{\text {th }}$ Century when a series of visits by naturalists and explorers started towards the then mountain kingdom of Sikkim Himalaya (Chaudhary 1951, Clarke 1876, Hara 1966, Hooker 1849, King \& Pantling 1889, Mehra \& Bir 1964, Rao 1963, Smith 1913, Smith \& Cave 1911). The ethnobotany of the area was first described by Atkinson (1882), followed by Biswas (1956), Puri \& Pandey (1980), Hajra \& Chakraborty (1981), Bennet (1983, 1985), Krishna \& Singh (1987), Rai \& Sharma (1994), Jana \& Chauhan (1999, 2000), Maiti et al. (2003), Dash et al. (2003), Pradhan \& Badola (2008). Despite all of this research, the ethnovetenary practices of the region remained unexplored. Modernization of Sikkim has increased cultural interaction with modern medicine, media and other cultural groups. This has resulted in a decrease in traditional knowledge and cultural practrices. Many areas of Sikkim remain isolated due to a lack of modern infrastructure there, and in these areas local tradtional knowledge persists. Keeping this in mind, we set out to document the prevalent knowledge being used by the local people of Sikkim. The aims of this project are the:

1. Investigation of traditional medicinal plant knowledge of Sikkim with special reference to livestock treatment.

2. Conservation of indigenous knowledge through documentation.

\section{Methods}

An ethnobotanical survey was conducted from March 2008 to June 2009 in 15 villages of Sikkim (Figure 1). After consultation with local people the sample villages, representing different geographical locations, climatic conditions, vegetation and major ethnic groups of the state were identified. Also included were Bhutia nomadic herdsmen, who were interviewed near Yumthang. A Transect Walk method of a Participatory Rural Appraisal (PRA) was adopted (Cunningham 2001). This method involves semistructured interviews and discussion with key-research participants such as, community elders, traditional healers, livestock owners and herdsmen. The interviews were held in Nepali.

The prevalent animal diseases, medicinal plants and other raw materials, methods of preparation and dosage of remedies used were recorded. Plant voucher specimens were collected with key informants in the areas where they normally collect medicinal plants, as part of the Transect Walk process. Plants were identified using Hajra and Verma (1996). Additional identification was done by matching voucher specimens with previously identified specimens held in local herbaria (BSHC, DD). Voucher specimens from this research have been deposited at Raw Materials Herbarium \& Museum Delhi (RHMD) and at the Department of Botany, N.R.E.C. College, Uttar Pradesh, India. A comparative assessment in the form of a literature review was also conducted to differentiate between new findings and similarities with past research (Tables 1, 2).

\section{Results}

A total of 46 people ( 30 men and 16 women), ranging from 30 to 70 years in age were interviewed. Key-research participants included members of the three main ethnic groups in Sikkim, Lepcha (9 men and 3 women), Bhutia ( 5 men and 5 women) and Nepali (16 men and 8 women).

Table 1. Plant species used in ethnoveterinary practices in Sikkim, India. Local names are given in the three main languages of the region, $(B)=$ Bhutia, $(L)=$ Lepcha, $(N)=$ Nepali.

\begin{tabular}{|l|l|l|l|l|l|}
\hline $\begin{array}{l}\text { Species name } \\
\text { and family }\end{array}$ & $\begin{array}{l}\text { Specimen } \\
\text { number }\end{array}$ & $\begin{array}{l}\text { Local } \\
\text { names }\end{array}$ & Distribution & $\begin{array}{l}\text { Ethnoveterinary } \\
\text { use (Present } \\
\text { study) }\end{array}$ & $\begin{array}{l}\text { Ethnoverinary use in } \\
\text { other parts of India } \\
\text { (Earlier studies) }\end{array}$ \\
\hline $\begin{array}{l}\text { Abies densa Griff. } \\
\text { (Pinaceae) }\end{array}$ & KAB17 & Ailey $(\mathrm{N})$ & $\begin{array}{l}\text { Temperate and } \\
\text { subalpine climate } \\
\text { from 2500 - } \\
4000 \mathrm{~m} \mathrm{ASL}\end{array}$ & $\begin{array}{l}\text { Fresh leaves } \\
\text { are crushed and } \\
\text { extract }(\approx 40 \mathrm{ml}) \\
\text { is given to cattle } \\
\text { to treat fever, loss } \\
\text { of appetite and } \\
\text { uneasy feeling. }\end{array}$ & \\
\hline
\end{tabular}




\begin{tabular}{|c|c|c|c|c|c|}
\hline $\begin{array}{l}\text { Species name } \\
\text { and family }\end{array}$ & $\begin{array}{l}\text { Specimen } \\
\text { number }\end{array}$ & $\begin{array}{l}\text { Local } \\
\text { names }\end{array}$ & Distribution & $\begin{array}{l}\text { Ethnoveterinary } \\
\text { use (Present } \\
\text { study) }\end{array}$ & $\begin{array}{l}\text { Ethnoverinary use in } \\
\text { other parts of India } \\
\text { (Earlier studies) }\end{array}$ \\
\hline $\begin{array}{l}\text { Acorus calamus } \\
\text { L. (Acoraceae) }\end{array}$ & KAB24 & $\begin{array}{l}\text { Bojo, } \\
\text { Bojho }(\mathrm{N})\end{array}$ & $\begin{array}{l}\text { Marshy or semi- } \\
\text { aquatic land in } \\
\text { between } 1700 \\
-2300 \text { m ASL }\end{array}$ & $\begin{array}{l}\text { Paste of root } \\
\text { and rhizome is } \\
\text { administered } \\
\text { twice daily to } \\
\text { treat indigestion } \\
\text { in livestock. } \\
\text { Rhizome paste is } \\
\text { used as ointment } \\
\text { on wounds. }\end{array}$ & $\begin{array}{l}\text { Roots are used as } \\
\text { anthelmintic and in } \\
\text { edema (Anjaria 2002); } \\
\text { hoof disease (Tiwari \& } \\
\text { Pande 2006; Pande et } \\
\text { al. 2007); removal of } \\
\text { external and internal } \\
\text { parasites, mouth blisters, } \\
\text { snake bite, hematuria, } \\
\text { wound (Pande et al. } \\
\text { 2007); dyspepsia } \\
\text { (Borthakur \& Sarma } \\
\text { 1996); appetizer (Jain } \\
\text { \& Srivastava 1999); } \\
\text { against flea (poultry), } \\
\text { snake bite, lice (Ghotage } \\
\text { \& Ramdas 2008). }\end{array}$ \\
\hline $\begin{array}{l}\text { Amomum } \\
\text { subulatum Roxb. } \\
\text { (Zingiberaceae) }\end{array}$ & KAB155 & Alaichi $(\mathrm{N})$ & $\begin{array}{l}\text { Cultivated in } \\
\text { between } 600 \\
-1800 \mathrm{~m} \text { ASL }\end{array}$ & $\begin{array}{l}\text { Oil extracted } \\
\text { from dry seeds } \\
\text { is applied on } \\
\text { eye-lids to allay } \\
\text { inflammation of } \\
\text { the eye. Paste of } \\
\text { seeds is applied } \\
\text { externally as } \\
\text { antidote for } \\
\text { scorpion-sting } \\
\text { and insect bites. } \\
\end{array}$ & \\
\hline $\begin{array}{l}\text { Artemisia vulgaris } \\
\text { L. (Asteraceae) }\end{array}$ & KAB128 & $\begin{array}{l}\text { Tetaypati, } \\
\text { Teil (N) }\end{array}$ & $\begin{array}{l}\text { Abundant } \\
\text { between } 1500 \\
-2500 \text { m ASL }\end{array}$ & $\begin{array}{l}\text { Leaves are } \\
\text { crushed and } \\
\text { extract is applied } \\
\text { externally on skin } \\
\text { to treat itching. } \\
\text { Fresh leaves are } \\
\text { cleaned, ground } \\
\text { and the sap } \\
\text { extracted. It is used } \\
\text { as nasal drop to } \\
\text { stop nose bleeding. }\end{array}$ & \\
\hline $\begin{array}{l}\text { Arundinaria } \\
\text { maling Gamble } \\
\text { (Poaceae) }\end{array}$ & KAB140 & Malingo $(\mathrm{N})$ & $\begin{array}{l}\text { Abundant from } \\
\text { 1800- } 2700 \\
\mathrm{~m} \mathrm{ASL}\end{array}$ & $\begin{array}{l}\text { Ash of the root is } \\
\text { mixed with a few } \\
\text { drops of mustard } \\
\text { oil and the paste is } \\
\text { applied externally } \\
\text { on ringworm. } \\
\text { Leaves of plant } \\
(\approx 20 \mathrm{gm}) \text { is } \\
\text { mixed with black } \\
\text { pepper }(\approx 5 \mathrm{gm}) \\
\text { and feed with } \\
\text { little salt to cattle } \\
\text { to treat diarrhea } \\
\text { and dysentery. }\end{array}$ & \\
\hline
\end{tabular}


Himalayas

\begin{tabular}{|c|c|c|c|c|c|}
\hline $\begin{array}{l}\text { Species name } \\
\text { and family }\end{array}$ & $\begin{array}{l}\text { Specimen } \\
\text { number }\end{array}$ & $\begin{array}{l}\text { Local } \\
\text { names }\end{array}$ & Distribution & $\begin{array}{l}\text { Ethnoveterinary } \\
\text { use (Present } \\
\text { study) } \\
\end{array}$ & $\begin{array}{l}\text { Ethnoverinary use in } \\
\text { other parts of India } \\
\text { (Earlier studies) }\end{array}$ \\
\hline $\begin{array}{l}\text { Asparagus } \\
\text { racemosus Willd. } \\
\text { (Asparagaceae) }\end{array}$ & KAB122 & $\begin{array}{l}\text { Satamuli } \\
\text { (N) }\end{array}$ & $\begin{array}{l}\text { From } 600 \text { - } \\
1800 \text { m ASL }\end{array}$ & $\begin{array}{l}\text { About } 100 \mathrm{gm} \\
\text { of root is mixed } \\
\text { with hay or grain } \\
\text { to feed the cattle } \\
\text { for fortnight to } \\
\text { increase milk-yield. }\end{array}$ & $\begin{array}{l}\text { Galactagogue, fatigue } \\
\text { (Anjaria 2002); Lactation } \\
\text { problems (Tiwari \& } \\
\text { Pande 2006; Kiruba } \\
\text { et al. 2006; Reddy \& } \\
\text { Raju 2000; Pande et } \\
\text { al. 2007); painful out- } \\
\text { growth below tongue, } \\
\text { hematuria, tympany, } \\
\text { flatulence, cuts, wounds, } \\
\text { demulcent, indigestion, } \\
\text { gastric trouble, skin } \\
\text { disease (Pande et } \\
\text { al. 2007); insect bite } \\
\text { (Jain \& Srivastava } \\
\text { 1999); galactagogue } \\
\text { (Ghotage \& Ramdas } \\
\text { 2008); arthritis } \\
\text { (Harsha et al. 2005). }\end{array}$ \\
\hline $\begin{array}{l}\text { Bauhinia } \\
\text { variegata L. } \\
\text { (Fabaceae) }\end{array}$ & KAB142 & Taki (N) & $\begin{array}{l}\text { Lower hills forest } \\
\text { of Terai region up } \\
\text { to } 1700 \mathrm{~m} \mathrm{ASL}\end{array}$ & $\begin{array}{l}\text { Fresh flowers } \\
\text { (about } 200 \mathrm{gm} \text { ) are } \\
\text { orally administered } \\
\text { thrice daily to cattle } \\
\text { to cure diarrhea } \\
\text { and dysentery. }\end{array}$ & $\begin{array}{l}\text { Internal injury (Pande } \\
\text { et al. 2007); foot and } \\
\text { mouth disease \& injury } \\
\text { (Pandey et al. 1999). }\end{array}$ \\
\hline $\begin{array}{l}\text { Bergenia } \\
\text { ciliata Sternb. } \\
\text { (Saxifragaceae) }\end{array}$ & KAB125 & $\begin{array}{l}\text { Pakhanbh- } \\
\text { ed, Bet }(N)\end{array}$ & $\begin{array}{l}\text { Between 1500- } \\
3000 \text { m ASL }\end{array}$ & $\begin{array}{l}\text { The root is crushed } \\
\text { and extract is } \\
\text { administered } \\
(\approx 40 \mathrm{ml}) \text { thrice } \\
\text { daily to cattle to } \\
\text { treat diarrhea } \\
\text { and dysentery. }\end{array}$ & $\begin{array}{l}\text { Mastitis (Tiwari \& } \\
\text { Pande 2006); weakness } \\
\text { and sickness (Singh } \\
\text { \& Kumar 2000). }\end{array}$ \\
\hline $\begin{array}{l}\text { Cannabis sativa L. } \\
\text { (Cannabaceae ) }\end{array}$ & KAB146 & $\begin{array}{l}\text { Ganja, } \\
\text { Bhang (N) }\end{array}$ & $\begin{array}{l}\text { Abundant on } \\
\text { lower hill forest } \\
\text { in Terai region }\end{array}$ & $\begin{array}{l}\text { Stem is cut into } \\
\text { small pieces and } \\
\text { fed to livestock to } \\
\text { treat inflammation. } \\
\text { Small pieces of } \\
\text { stem are mixed } \\
\text { with fodder to feed } \\
\text { cattle as a tonic. }\end{array}$ & $\begin{array}{l}\text { Stomachache (Tiwari \& } \\
\text { Pande 2006; Samal et } \\
\text { al. 2003); stomachache, } \\
\text { mouth disease, } \\
\text { dysentery, rheumatism, } \\
\text { piles, retention of } \\
\text { placenta, eczema, } \\
\text { internal injury, sprain, } \\
\text { flatulence, indigestion, } \\
\text { bone fracture, wound, } \\
\text { bleeding, expel } \\
\text { leeches (Pande et } \\
\text { al. 2007); dysentery } \\
\text { (Mishra et al. 1996); } \\
\text { diarrhea, dysentery, } \\
\text { increase strength } \\
\text { and vigour (Ghotage } \\
\text { \& Ramdas 2008). }\end{array}$ \\
\hline
\end{tabular}




\begin{tabular}{|c|c|c|c|c|c|}
\hline $\begin{array}{l}\text { Species name } \\
\text { and family }\end{array}$ & $\begin{array}{l}\text { Specimen } \\
\text { number }\end{array}$ & $\begin{array}{l}\text { Local } \\
\text { names }\end{array}$ & Distribution & $\begin{array}{l}\text { Ethnoveterinary } \\
\text { use (Present } \\
\text { study) }\end{array}$ & $\begin{array}{l}\text { Ethnoverinary use in } \\
\text { other parts of India } \\
\text { (Earlier studies) }\end{array}$ \\
\hline $\begin{array}{l}\text { Celastrus } \\
\text { paniculatus Willd. } \\
\text { (Celastraceae ) }\end{array}$ & KAB158 & Ruglin (L) & $\begin{array}{l}\text { Tropical and } \\
\text { subtropical } \\
\text { climate up to } \\
1800 \text { m ASL }\end{array}$ & $\begin{array}{l}\text { About } 50 \mathrm{gm} \text { of } \\
\text { leaves are given } \\
\text { twice daily to } \\
\text { cattle to treat } \\
\text { loss of appetite. }\end{array}$ & $\begin{array}{l}\text { Restlessness, } \\
\text { hyperactivity, morbidity } \\
\text { (Anjaria 2002). }\end{array}$ \\
\hline $\begin{array}{l}\text { Centella asiatica } \\
\text { (L.) Urb. } \\
\text { (Apiaceae) }\end{array}$ & KAB104 & $\begin{array}{l}\text { Ghod } \\
\text { Tapre, } \\
\text { Bhram } \\
\text { Jhar }(\mathrm{N})\end{array}$ & $\begin{array}{l}\text { Moist places in } \\
\text { between 1300- } \\
2000 \text { m ASL }\end{array}$ & $\begin{array}{l}\text { About } 20 \text { gm of } \\
\text { plant is crushed } \\
\text { and administered } \\
\text { thrice daily to } \\
\text { livestock to cure } \\
\text { urinary disorders } \\
\text { such as little } \\
\text { and frequent } \\
\text { urine pass. }\end{array}$ & $\begin{array}{l}\text { Restlessness, } \\
\text { hyperactive, morbility } \\
\text { (Anjaria 2002); sunstroke } \\
\text { (Tiwari \& Pande 2006); } \\
\text { dyspepsia (Borthakur } \\
\text { \& Sarma 1996); ulcer, } \\
\text { pneumonia, dyspepsia, } \\
\text { urinary disorder, } \\
\text { galactagogue (Jain } \\
\text { \& Srivastava 1999); } \\
\text { galactagogue, gives } \\
\text { strength (Ghotage } \\
\text { \& Ramdas 2008). }\end{array}$ \\
\hline $\begin{array}{l}\text { Cissampelos } \\
\text { pareira L. } \\
\text { (Menispermaceae) }\end{array}$ & KAB106 & \begin{tabular}{|l|} 
Tamshaprip \\
(L), Batul \\
Pati (N)
\end{tabular} & $\begin{array}{l}\text { Temperate } \\
\text { climatic condition } \\
\text { from } 1600- \\
2500 \mathrm{~m} \mathrm{ASL}\end{array}$ & $\begin{array}{l}\text { Paste of root is } \\
\text { applied externally } \\
\text { as antidote on } \\
\text { insect bite and } \\
\text { scorpion sting. The } \\
\text { root is crushed } \\
\text { and extract is } \\
\text { given twice daily } \\
\text { to cattle to treat } \\
\text { blood in urine. }\end{array}$ & $\begin{array}{l}\text { Swelling of abdomen } \\
\text { due to flatulence } \\
\text { (Bandyopadhyay \& } \\
\text { Mukherjee 2005); fever } \\
\text { (Jain \& Srivastava } \\
\text { 1999); jaundice (Jain } \\
\text { \& Srivastava 2003). }\end{array}$ \\
\hline $\begin{array}{l}\text { Costus speciosus } \\
\text { (J. König) Sm. } \\
\text { (Costaceae) }\end{array}$ & KAB135 & $\begin{array}{l}\text { Bet Lauree } \\
(\mathrm{N})\end{array}$ & $\begin{array}{l}\text { Tropical and } \\
\text { subtropical } \\
\text { climate up to } \\
1700 \text { m ASL }\end{array}$ & $\begin{array}{l}\text { Rhizome powder } \\
(\approx 20 \mathrm{gm}) \text { is given } \\
\text { twice daily to cattle } \\
\text { to treat fever and } \\
\text { inflammation. }\end{array}$ & $\begin{array}{l}\text { Wound (Bandyopadhyay } \\
\text { \& Mukherjee 2005); } \\
\text { Injuries and bruises } \\
\text { (Ghotage \& Ramdas } \\
\text { 2008); jaundice (Jain } \\
\text { \& Srivastava 2003). }\end{array}$ \\
\hline $\begin{array}{l}\text { Drymaria cordata } \\
\text { (L.) Willd. ex } \\
\text { Roem. \& Schult. } \\
\text { (Caryophyllaceae) }\end{array}$ & KAB19 & Abhijal (N) & Up to $2100 \mathrm{~m} \mathrm{ASL}$ & $\begin{array}{l}\text { Plant paste is } \\
\text { applied externally } \\
\text { on fractured bone } \\
\text { and bandaged with } \\
\text { the help of cotton } \\
\text { cloth. After } 20-25 \\
\text { days bandage } \\
\text { is removed. } \\
\text { About } 100 \mathrm{gm} \text { of } \\
\text { plant is boiled in } \\
\text { water to prepare } \\
\text { decoction. This } \\
\text { decoction is } \\
\text { administered thrice } \\
\text { daily to animal to } \\
\text { treat mouth ulcer. }\end{array}$ & \\
\hline
\end{tabular}


Himalayas

\begin{tabular}{|c|c|c|c|c|c|}
\hline $\begin{array}{l}\text { Species name } \\
\text { and family }\end{array}$ & $\begin{array}{l}\text { Specimen } \\
\text { number }\end{array}$ & $\begin{array}{l}\text { Local } \\
\text { names }\end{array}$ & Distribution & $\begin{array}{l}\text { Ethnoveterinary } \\
\text { use (Present } \\
\text { study) }\end{array}$ & $\begin{array}{l}\text { Ethnoverinary use in } \\
\text { other parts of India } \\
\text { (Earlier studies) }\end{array}$ \\
\hline $\begin{array}{l}\text { Elatostema } \\
\text { ficoides Wedd. } \\
\text { (Urticaceae) }\end{array}$ & KAB64 & $\begin{array}{l}\text { Chiplu, } \\
\text { Chiplay (N) }\end{array}$ & $\begin{array}{l}\text { Between } 1200 \\
-2500 \text { m ASL }\end{array}$ & $\begin{array}{l}\text { About } 20-50 \mathrm{gm} \\
\text { of plant is crushed } \\
\text { and given twice } \\
\text { daily to livestock } \\
\text { to treat fever. }\end{array}$ & \\
\hline $\begin{array}{l}\text { Embelia } \\
\text { ribes Burm. f. } \\
\text { (Primulaceae) }\end{array}$ & KAB98 & $\begin{array}{l}\text { Buibidans, } \\
\text { Pierlahara } \\
\text { (N), Sangrik } \\
\text { Asumbu (L) }\end{array}$ & $\begin{array}{l}\text { Tropical climate } \\
\text { on lower hill forest } \\
\text { in Terai region }\end{array}$ & $\begin{array}{l}\text { About } 200 \text { gm of } \\
\text { fruits are crushed } \\
\text { and given to pigs } \\
\text { for two days to } \\
\text { kill tapeworm. } \\
\text { Fruits are also } \\
\text { used as appetizer } \\
\text { for cattle, about } \\
50 \text { gm of fruit } \\
\text { is crushed and } \\
\text { administered daily } \\
\text { until complete cure } \\
\text { of the disease. }\end{array}$ & $\begin{array}{l}\text { Fever, stomach worms } \\
\text { (Jain \& Srivastava } \\
\text { 1999); sprains, worms } \\
\text { in calves (Ghotage } \\
\text { \& Ramdas 2008). }\end{array}$ \\
\hline $\begin{array}{l}\text { Equisetum debile } \\
\text { Roxb. ex Vaucher } \\
\text { (Equisetaceae) }\end{array}$ & KAB70 & $\begin{array}{l}\text { Kurkure } \\
\text { Ghans (N) }\end{array}$ & $\begin{array}{l}\text { Common, in } \\
\text { shaded or open } \\
\text { water logged } \\
\text { areas, or along } \\
\text { streams from } \\
1000-2500 \\
\text { m ASL }\end{array}$ & $\begin{array}{l}\text { Paste of stem } \\
\text { shoot is applied } \\
\text { externally on injury } \\
\text { to stop bleeding. } \\
\text { About } 20 \text { gm of } \\
\text { plant is crushed } \\
\text { and given twice } \\
\text { daily with jaggery } \\
\text { to livestock to treat } \\
\text { blood in urine and } \\
\text { blood secretion } \\
\text { from vagina. }\end{array}$ & \\
\hline $\begin{array}{l}\text { Fragaria nubicola } \\
\text { (Hook. f.) Lindl. } \\
\text { ex Lacaita } \\
\text { (Rosaceae) }\end{array}$ & KAB134 & $\begin{array}{l}\text { Bhui } \\
\text { Ainselu (N) }\end{array}$ & $\begin{array}{l}\text { Temperate } \\
\text { climatic condition } \\
\text { from } 1800- \\
4000 \text { m ASL }\end{array}$ & $\begin{array}{l}\text { About } 50 \text { gm of } \\
\text { pounded leaves } \\
\text { and fruits are } \\
\text { administered } \\
\text { with lukewarm } \\
\text { water to cattle } \\
\text { to treat diarrhea } \\
\text { and dysentery. } \\
\text { Leaves and fruits } \\
\text { are also used as } \\
\text { diuretic for cattle, } \\
\text { about } 50 \text { gm is } \\
\text { administered twice } \\
\text { daily until cure. }\end{array}$ & $\begin{array}{l}\text { External parasite } \\
\text { (Pande et al. 2007). }\end{array}$ \\
\hline $\begin{array}{l}\text { Holarrhena } \\
\text { antidysenterica } \\
\text { (L.) Wall. ex A. DC. } \\
\text { (Apocynaceae) }\end{array}$ & KAB48 & $\begin{array}{l}\text { Indrajow, } \\
\text { Kurchi, } \\
\text { Aulay } \\
\text { Khirrn (N) }\end{array}$ & $\begin{array}{l}\text { Lower hills forest } \\
\text { of Terai region up } \\
\text { to } 500 \text { m ASL }\end{array}$ & $\begin{array}{l}\text { About } 20 \text { gm } \\
\text { stem bark powder } \\
\text { or decoction is } \\
\text { given thrice daily } \\
\text { to livestock to } \\
\text { treat constipation, } \\
\text { problems during } \\
\text { stool passing } \\
\text { and dysentery. }\end{array}$ & $\begin{array}{l}\text { Constipation, diarrhea, } \\
\text { cold and cough, } \\
\text { anthelmintic (Anjaria } \\
\text { 2002); Anthrax (Sikarwar } \\
\text { 1996); Appetizer (Jain } \\
\text { \& Srivastava 1999). }\end{array}$ \\
\hline
\end{tabular}




\begin{tabular}{|c|c|c|c|c|c|}
\hline $\begin{array}{l}\text { Species name } \\
\text { and family }\end{array}$ & $\begin{array}{l}\text { Specimen } \\
\text { number }\end{array}$ & $\begin{array}{l}\text { Local } \\
\text { names }\end{array}$ & Distribution & $\begin{array}{l}\text { Ethnoveterinary } \\
\text { use (Present } \\
\text { study) }\end{array}$ & $\begin{array}{l}\text { Ethnoverinary use in } \\
\text { other parts of India } \\
\text { (Earlier studies) }\end{array}$ \\
\hline $\begin{array}{l}\text { Lecanthus } \\
\text { peduncularis (Wall. } \\
\text { Ex Royle) Wedd. } \\
\text { (Urticaceae) }\end{array}$ & KAB50 & $\begin{array}{l}\text { Kala Jhar } \\
(\mathrm{N})\end{array}$ & $\begin{array}{l}\text { In between 1500- } \\
3500 \text { m ASL }\end{array}$ & $\begin{array}{l}\text { The leaf paste } \\
\text { is applied as } \\
\text { ointment on cuts } \\
\text { and wounds. }\end{array}$ & $\begin{array}{l}\text { Galactogogue (Jain } \\
\text { \& Srivastava 1999). }\end{array}$ \\
\hline $\begin{array}{l}\text { Lycopodium } \\
\text { clavatum L. } \\
\text { (Lycopodiaceae) }\end{array}$ & KAB71 & $\begin{array}{l}\text { Nagebeli } \\
\text { (N) }\end{array}$ & $\begin{array}{l}\text { Very common; } \\
\text { forming mat } \\
\text { over grasses, } \\
\text { found from 1800- } \\
2400 \text { m ASL }\end{array}$ & $\begin{array}{l}\text { About } 20 \mathrm{gm} \\
\text { of plant is } \\
\text { administered orally } \\
\text { for treatment of } \\
\text { muscle contraction } \\
\text { in cattle. }\end{array}$ & \\
\hline $\begin{array}{l}\text { Mallotus } \\
\text { philippinensis } \\
\text { Muell.-Arg. } \\
\text { (Euphorbiaceae ) }\end{array}$ & KAB73 & $\begin{array}{l}\text { Numboong- } \\
\text { kor, Purva, } \\
\text { Tukla (L); } \\
\text { Sinduri (N) }\end{array}$ & $\begin{array}{l}\text { Lower hill forest } \\
\text { in Terai region. }\end{array}$ & $\begin{array}{l}\text { About } 50 \mathrm{gm} \text { seeds } \\
\text { are administered } \\
\text { to pigs along-with } \\
\text { food to kill intestinal } \\
\text { worms. Seeds } \\
\text { are crushed and } \\
\text { applied externally } \\
\text { to cure wound, } \\
\text { injuries and } \\
\text { skin infection. }\end{array}$ & $\begin{array}{l}\text { Anthelmintics, anorexia, } \\
\text { fatigue (Anjaria 2002); } \\
\text { diarrhea and dysentery } \\
\text { (Singh \& Kumar 2000). }\end{array}$ \\
\hline $\begin{array}{l}\text { Mentha viridis (L.) } \\
\text { L. (Lamiaceae) }\end{array}$ & KAB138 & $\begin{array}{l}\text { Mentha, } \\
\text { Babri (N) }\end{array}$ & $\begin{array}{l}\text { Cultivated in } \\
\text { subtropical } \\
\text { climatic condition }\end{array}$ & $\begin{array}{l}\text { About } 200 \mathrm{gm} \\
\text { leaves are crushed } \\
\text { with water and } \\
\text { boiled to prepare } \\
\text { decoction. Two } \\
\text { cups of decoction } \\
\text { is given thrice daily } \\
\text { to treat mild fever. }\end{array}$ & \\
\hline $\begin{array}{l}\text { Picrorhiza kurroa } \\
\text { Royle ex Benth. } \\
\text { (Scrophulariaceae) }\end{array}$ & KAB109 & Kutki $(\mathrm{N})$ & $\begin{array}{l}\text { Subalpine and } \\
\text { alpine climatic } \\
\text { condition from } \\
2100-4200 \\
\text { m ASL }\end{array}$ & $\begin{array}{l}\text { About } 100 \mathrm{gm} \\
\text { dried root is mixed } \\
\text { with stem bark } \\
\text { of Azadirachta } \\
\text { indica A. Juss. } \\
\text { in a ratio of } 4: 1 \text {. } \\
\text { These are boiled } \\
\text { in water to prepare } \\
\text { decoction. The } \\
\text { decoction is given } \\
\text { thrice daily to treat } \\
\text { cold and fever. } \\
\text { Root is ground, } \\
\text { boiled in water to } \\
\text { prepare decoction. } \\
\text { About } 100 \mathrm{ml} \text { of } \\
\text { root decoction is } \\
\text { given to animal } \\
\text { as appetizer. }\end{array}$ & $\begin{array}{l}\text { Tonsil (Tiwari \& Pande } \\
\text { 2006); loss of appetite, } \\
\text { fever (Tiwari \& Pande } \\
\text { 2004); digestive troubles, } \\
\text { dysentery, alimentary } \\
\text { disorders, intestinal } \\
\text { worm, diarrhea (Pande } \\
\text { et al. 2007); wound, } \\
\text { hypocaemia (Jain \& } \\
\text { Srivastava 1999). }\end{array}$ \\
\hline $\begin{array}{l}\text { Plantago major L. } \\
\text { (Plantaginaceae) }\end{array}$ & KAB29 & $\begin{array}{l}\text { Nasha } \\
\text { Jhar }(\mathrm{N})\end{array}$ & $\begin{array}{l}\text { Frequently grow } \\
\text { along roadsides } \\
\text { from } 1200- \\
2600 \text { m ASL }\end{array}$ & $\begin{array}{l}\text { Paste made from } \\
\text { root is applied } \\
\text { externally on cuts } \\
\text { and wounds for } \\
\text { quick healing. }\end{array}$ & \\
\hline
\end{tabular}


Himalayas

\begin{tabular}{|c|c|c|c|c|c|}
\hline $\begin{array}{l}\text { Species name } \\
\text { and family }\end{array}$ & $\begin{array}{l}\text { Specimen } \\
\text { number }\end{array}$ & $\begin{array}{l}\text { Local } \\
\text { names }\end{array}$ & Distribution & $\begin{array}{l}\text { Ethnoveterinary } \\
\text { use (Present } \\
\text { study) }\end{array}$ & $\begin{array}{l}\text { Ethnoverinary use in } \\
\text { other parts of India } \\
\text { (Earlier studies) }\end{array}$ \\
\hline $\begin{array}{l}\text { Rauvolfia } \\
\text { serpentina (L.) } \\
\text { Benth. ex Kurz. } \\
\text { (Apocynaceae) }\end{array}$ & KAB26 & $\begin{array}{l}\text { Sarpgan- } \\
\text { dha }(N)\end{array}$ & $\begin{array}{l}\text { Lower hills forest } \\
\text { of Terai region up } \\
\text { to } 600 \mathrm{~m} \mathrm{ASL}\end{array}$ & $\begin{array}{l}\text { About } 20 \text { gm } \\
\text { powder of root bark } \\
\text { is administered } \\
\text { orally as antidote } \\
\text { to snake-bite. }\end{array}$ & $\begin{array}{l}\text { The root is used as } \\
\text { nervine tonic, eye } \\
\text { problem, diarrhea (Jain } \\
\text { \& Srivastava 1999). }\end{array}$ \\
\hline $\begin{array}{l}\text { Rhododendron } \\
\text { arboreum Sm. } \\
\text { (Ericaceae) }\end{array}$ & KAB119 & $\begin{array}{l}\text { Etok (L); } \\
\text { Ghonas, } \\
\text { Gurangs, } \\
\text { Guras, } \\
\text { Lal-Gurans, } \\
\text { Lalli- } \\
\text { Gurans (N) }\end{array}$ & $\begin{array}{l}\text { Temperate and } \\
\text { upper hill forest } \\
\text { from } 1500- \\
3300 \text { m ASL }\end{array}$ & $\begin{array}{l}\text { About } 20 \text { - 50gm } \\
\text { flowers are crushed } \\
\text { with water and } \\
\text { administered twice } \\
\text { a day to livestock } \\
\text { to treat diarrhea } \\
\text { and dysentery. }\end{array}$ & $\begin{array}{l}\text { Leaves are used in } \\
\text { removal of external } \\
\text { parasites (Tiwari \& } \\
\text { Pande 2006; Pande } \\
\text { et al. 2007). }\end{array}$ \\
\hline $\begin{array}{l}\text { Rhododendron } \\
\text { campanulatum } \\
\text { D.Don } \\
\text { (Ericaceae) } \\
\text { Note: However, } \\
\text { it is reported that } \\
\text { young buds and } \\
\text { leaves of the plant } \\
\text { are poisonous to } \\
\text { cattle (Anonymous } \\
\text { 2004). }\end{array}$ & KAB120 & $\begin{array}{l}\text { Bargi }(\mathrm{B}) ; \\
\text { Nilo } \\
\text { Chimal, } \\
\text { Cheriala, } \\
\text { Teotosa, } \\
\text { Gurans (N) }\end{array}$ & $\begin{array}{l}\text { Usually in } \\
\text { gregarious } \\
\text { patches in upper } \\
\text { hill forest from } \\
2700-4400 \text { m ASL }\end{array}$ & $\begin{array}{l}\text { Leaves and twigs } \\
\text { are mixed with } \\
\text { leaves of tobacco } \\
\text { (Nicotiana tabacum } \\
\text { L.) in ratio of } 3: 1 . \\
\text { They are crushed } \\
\text { together and about } \\
10-15 \text { gm of paste } \\
\text { is administered } \\
\text { orally twice a day } \\
\text { with lukewarm } \\
\text { water to cattle to } \\
\text { treat chronic fever. }\end{array}$ & $\begin{array}{l}\text { Leaves are used as goat } \\
\text { poison at Uttranchal } \\
\text { (Pande et al. 2007). }\end{array}$ \\
\hline $\begin{array}{l}\text { Rubia cordifolia L. } \\
\text { (Rubiaceae) }\end{array}$ & KAB95 & $\begin{array}{l}\text { Vhyem, } \\
\text { Vhyeni (L); } \\
\text { Soth (B); } \\
\text { Manjito (N) }\end{array}$ & $\begin{array}{l}\text { Climber on } \\
\text { shrubs From } \\
1200-2500 \text { m ASL }\end{array}$ & $\begin{array}{l}\text { Root paste is used } \\
\text { as ointment to } \\
\text { treat skin infection. } \\
\text { About } 100 \text { gm of } \\
\text { roots are cut into } \\
\text { small pieces and } \\
\text { boiled to prepare } \\
\text { a decoction. } \\
\text { The decoction is } \\
\text { administered ( } \approx \\
100 \text { ml) to cattle } \\
\text { after delivery for } \\
\text { quick recovery. }\end{array}$ & $\begin{array}{l}\text { Lactation, swelling, } \\
\text { sunstroke, skin disease } \\
\text { (Pande et al. 2007). }\end{array}$ \\
\hline $\begin{array}{l}\text { Rubus ellipticus } \\
\text { Sm. (Rosaceae) }\end{array}$ & KAB78 & $\begin{array}{l}\text { Ainselu, } \\
\text { Asayloo } \\
(\mathrm{N}) \text {; } \\
\text { Kashyem } \\
\text { (L) }\end{array}$ & $\begin{array}{l}\text { Abundantly } \\
\text { in subtropical } \\
\text { and temperate } \\
\text { areas from } 600 \text { - } \\
2100 \text { m ASL }\end{array}$ & $\begin{array}{l}\text { About } 50 \text { gm of } \\
\text { new tender leaves } \\
\text { and/or young } \\
\text { stem shoots are } \\
\text { pounded and } \\
\text { administered to } \\
\text { livestock to treat } \\
\text { cold and fever. } \\
\text { Root paste is } \\
\text { applied externally } \\
\text { on wound for } \\
\text { quick recovery. }\end{array}$ & $\begin{array}{l}\text { Hematuria (Pande } \\
\text { et al. 2007). }\end{array}$ \\
\hline
\end{tabular}




\begin{tabular}{|c|c|c|c|c|c|}
\hline $\begin{array}{l}\text { Species name } \\
\text { and family }\end{array}$ & $\begin{array}{l}\text { Specimen } \\
\text { number }\end{array}$ & $\begin{array}{l}\text { Local } \\
\text { names }\end{array}$ & Distribution & $\begin{array}{l}\text { Ethnoveterinary } \\
\text { use (Present } \\
\text { study) }\end{array}$ & $\begin{array}{l}\text { Ethnoverinary use in } \\
\text { other parts of India } \\
\text { (Earlier studies) }\end{array}$ \\
\hline $\begin{array}{l}\text { Sinopodophyllum } \\
\text { hexandrum } \\
\text { (Royle) T.S. Ying } \\
\text { (Berberidaceae) }\end{array}$ & KAB116 & $\begin{array}{l}\text { Papari, } \\
\text { Panchpatey } \\
(\mathrm{N})\end{array}$ & $\begin{array}{l}\text { Interior range of } \\
\text { the Himalayas at } \\
\text { altitude of } 2700 \\
-4200 \mathrm{~m} \mathrm{ASL}\end{array}$ & $\begin{array}{l}\text { The root is crushed } \\
\text { and applied } \\
\text { externally on hoof } \\
\text { to treat infection. } \\
\text { Rhizome is } \\
\text { crushed and } \\
\text { boiled in water to } \\
\text { prepare decoction. } \\
\text { One bamboo cup } \\
\text { of decoction is } \\
\text { administered twice } \\
\text { a day to cattle to } \\
\text { treat indigestion. }\end{array}$ & \\
\hline $\begin{array}{l}\text { Swertia chirata } \\
\text { (Wall.) C.B. Clarke } \\
\text { (Gentianaceae) }\end{array}$ & KAB49 & $\begin{array}{l}\text { Chirowto } \\
(\mathrm{N})\end{array}$ & $\begin{array}{l}\text { Usually found } \\
\text { at shady places } \\
\text { from } 1600 \text { - } \\
2600 \text { m ASL }\end{array}$ & $\begin{array}{l}\text { Fresh plant }(\approx \\
100 \text { gm) is cut } \\
\text { into small pieces } \\
\text { and decoction } \\
\text { is prepared. } \\
\text { This is later } \\
\text { filtered through } \\
\text { a cotton cloth } \\
\text { and administered } \\
\text { orally to cattle } \\
\text { to treat fever. }\end{array}$ & $\begin{array}{l}\text { Tonic (Anjaria 2002); } \\
\text { hoof disease, eye } \\
\text { disease (Pande et al. } \\
\text { 2007); fever (Pandey } \\
\text { et al. 1999). }\end{array}$ \\
\hline $\begin{array}{l}\text { Terminalia bellirica } \\
\text { (Gaertn.) Rox. } \\
\text { (Combretaceae) }\end{array}$ & KAB54 & Barra $(\mathrm{N})$ & $\begin{array}{l}\text { Lower hill forests } \\
\text { of Terai region up } \\
\text { to } 1000 \mathrm{~m} \mathrm{ASL}\end{array}$ & $\begin{array}{l}\text { About } 100 \text { gm of } \\
\text { fruit are crushed } \\
\text { with water and } \\
\text { boiled to prepare } \\
\text { decoction. It is } \\
\text { administered thrice } \\
\text { daily to livestock } \\
\text { for treatment } \\
\text { of diarrhea and } \\
\text { dysentery. }\end{array}$ & $\begin{array}{l}\text { Constipation, naval } \\
\text { swelling, cold and } \\
\text { cough (Anjaria 2002); } \\
\text { diarrhea (Pande et } \\
\text { al. 2007); wound } \\
\text { (Harsha et al. 2005). }\end{array}$ \\
\hline $\begin{array}{l}\text { Terminalia } \\
\text { chebula Retz. } \\
\text { (Combretaceae) }\end{array}$ & KAB59 & $\begin{array}{l}\text { Harra }(\mathrm{N}) \text {; } \\
\text { Selim } \\
\text { Pot }(\mathrm{L})\end{array}$ & $\begin{array}{l}\text { Lower hills forest } \\
\text { of Terai region up } \\
\text { to } 1000 \mathrm{~m} \mathrm{ASL}\end{array}$ & $\begin{array}{l}\text { About } 100 \text { gm of } \\
\text { fruit and/or bark } \\
\text { are crushed and } \\
\text { boiled in water to } \\
\text { prepare decoction. } \\
\text { The decoction } \\
\text { is administered } \\
\text { thrice daily with } \\
\text { small amounts of } \\
\text { rock salt to cattle } \\
\text { to treat diarrhea } \\
\text { and dysentery. }\end{array}$ & $\begin{array}{l}\text { Constipation, diarrhea, } \\
\text { indigestion, anorexia, } \\
\text { flatulence, naval } \\
\text { swelling, cold and } \\
\text { cough, epistaxis, wound, } \\
\text { restlessness (Anjaria } \\
\text { 2002); rickets (Samal } \\
\text { et al. 2003; Pande et } \\
\text { al. 2007); constipation } \\
\text { (Singh \& Kumar 2000); } \\
\text { skin infection, diarrhea } \\
\text { (Pande et al. 2007). }\end{array}$ \\
\hline $\begin{array}{l}\text { Urtica dioica L. } \\
\text { (Utricaceae) }\end{array}$ & KAB93 & $\begin{array}{l}\text { Sisnu (N); } \\
\text { Surang (L) }\end{array}$ & $\begin{array}{l}\text { Abundant in } \\
\text { temperate zone } \\
\text { between } 1000 \\
-2200 \mathrm{~m} \mathrm{ASL} \\
\text { on middle hills } \\
\text { along roadsides }\end{array}$ & $\begin{array}{l}\text { Paste of roots is } \\
\text { applied externally } \\
\text { to bone fractures } \\
\text { with a cotton cloth. } \\
\text { About } 30 \text { gm young } \\
\text { leaves are mixed } \\
\text { with ripened }\end{array}$ & $\begin{array}{l}\text { Galactagogue, increase } \\
\text { egg production in } \\
\text { poultry (Deshpande } \\
\text { 2006); Bone fracture } \\
\text { (Tiwari \& Pande 2004); } \\
\text { galactagogue (Borthakur } \\
\text { \& Sarma 1996). }\end{array}$ \\
\hline
\end{tabular}


Bharati \& Sharma - Plants used as Ethnoveterinary Medicines in Sikkim Himalayas

\begin{tabular}{|c|c|c|c|c|c|}
\hline $\begin{array}{l}\text { Species name } \\
\text { and family }\end{array}$ & $\begin{array}{l}\text { Specimen } \\
\text { number }\end{array}$ & $\begin{array}{l}\text { Local } \\
\text { names }\end{array}$ & Distribution & \begin{tabular}{|l} 
Ethnoveterinary \\
use (Present \\
study)
\end{tabular} & $\begin{array}{l}\text { Ethnoverinary use in } \\
\text { other parts of India } \\
\text { (Earlier studies) }\end{array}$ \\
\hline $\begin{array}{l}\text { Urtica dioica L. } \\
\text { (Utricaceae) } \\
\text { con't }\end{array}$ & & & $\begin{array}{l}\text { and edges of } \\
\text { cultivation. }\end{array}$ & $\begin{array}{l}\text { tamarind fruit } \\
\text { (Tamarindus indica } \\
\text { L.) in a ratio of } \\
2: 1 \text { and crushed } \\
\text { together. The } \\
\text { preparation is } \\
\text { administered }(\approx \\
50 \mathrm{gm}) \text { to cattle to } \\
\text { increase milk yield. }\end{array}$ & \\
\hline $\begin{array}{l}\text { Zingiber } \\
\text { officinale Roscoe } \\
\text { (Zingiberaceae) }\end{array}$ & KAB66 & Aduwa $(\mathrm{N})$ & $\begin{array}{l}\text { Cultivated up to } \\
1200 \mathrm{~m} \mathrm{ASL}\end{array}$ & $\begin{array}{l}\text { About 20gm of } \\
\text { rhizome of plant is } \\
\text { mixed with fruits } \\
\text { of long pepper } \\
\text { (Piper longum L.) } \\
\text { in a ratio of } 4: 1 . \\
\text { These are crushed } \\
\text { together and } \\
\text { given with Jaggery } \\
\text { to cattle for } \\
\text { treatment of cold. } \\
\text { About } 20 \text { gm } \\
\text { rhizomes are } \\
\text { ground and the } \\
\text { extract is fed to } \\
\text { animal in the } \\
\text { morning and } \\
\text { evening with } \\
\text { lukewarm rice wash } \\
\text { to treat cough. }\end{array}$ & $\begin{array}{l}\text { Indigestion, anorexia, } \\
\text { flatulence, naval } \\
\text { swelling, cold and cough, } \\
\text { restlessness (Anjaria } \\
\text { 2002); constipation, } \\
\text { food poisoning (Tiwari \& } \\
\text { Pande 2006); swelling } \\
\text { (Tiwari \& Pande 2004); } \\
\text { cough, fever, indigestion, } \\
\text { loss of hunger (Mishra et } \\
\text { al. 1996); conjunctivitis, } \\
\text { ephemeral fever, } \\
\text { pneumonia, bloat, } \\
\text { fever, hemorrhagic } \\
\text { septicaemia, poisoning, } \\
\text { skin allergy (Ghotage } \\
\text { \& Ramdas 2008). }\end{array}$ \\
\hline
\end{tabular}

Table 2. Comparative study of plant species used in ethnoveterinary and human medicine in Sikkim, India.

\begin{tabular}{|l|l|l|l|l|}
\hline \multirow{2}{*}{ Species } & \multicolumn{3}{|l|}{ Veterinary medicine (Present study) } & Human medicine (Earlier studies) \\
\cline { 2 - 5 } & Part & Use & Part & Use \\
\hline Abies densa Griff. & Leaves & Fever and anorexia & Leaves & $\begin{array}{l}\text { Stomach ache, fever } \\
\text { (Pradhan \& Badola 2008) }\end{array}$ \\
\hline Acorus calamus L. & Roots, rhizomes & Indigestion, wound & Roots/rhizome & $\begin{array}{l}\text { Dysentery, skin disease } \\
\text { (Dash et al. 2003); fever, } \\
\text { skin disease (Rai \& } \\
\text { Sharma 1994); Itching } \\
\text { (Jana \& Chauhan 2000); } \\
\text { skin disease, fever, cough, } \\
\text { speech clarity (Pradhan } \\
\text { \& Badola 2008) }\end{array}$ \\
\hline Artemisia vulgaris L. & Leaves & & & $\begin{array}{l}\text { Nose bleeding, allergy, } \\
\text { mouth ulcer (Pradhan } \\
\text { \& Badola 2008) }\end{array}$ \\
\hline Bauhinia variegata L. & Flowers & Itching, nose bleeding & Leaves & $\begin{array}{l}\text { Ulcer, piles (Pradhan } \\
\text { \& Badola 2008) }\end{array}$ \\
\cline { 3 - 5 } & & Diarrhea, dysentery & Dried buds & $\begin{array}{l}\text { Tonic (Pradhan \& } \\
\text { Badola 2008) }\end{array}$ \\
\cline { 3 - 5 } & & Bark & \\
\hline
\end{tabular}




\begin{tabular}{|c|c|c|c|c|}
\hline \multirow[t]{2}{*}{ Species } & \multicolumn{2}{|c|}{ Veterinary medicine (Present study) } & \multicolumn{2}{|c|}{ Human medicine (Earlier studies) } \\
\hline & Part & Use & Part & Use \\
\hline $\begin{array}{l}\text { Bergenia ciliate } \\
\text { Sternb. }\end{array}$ & Roots & Diarrhea, dysentery & Rhizomes & $\begin{array}{l}\text { Diarrhea, vomiting, fever, } \\
\text { cough, pulmonary affections } \\
\text { (Rai \& Sharma, 1994) }\end{array}$ \\
\hline Cannabis sativa $\mathrm{L}$. & Stem & Inflammation, tonic & Seeds & $\begin{array}{l}\text { Body pain (Pradhan } \\
\text { \& Badola 2008) }\end{array}$ \\
\hline $\begin{array}{l}\text { Costus speciosus } \\
\text { (J. König) Sm. }\end{array}$ & Rhizomes & Fever, inflammation & Rhizomes & $\begin{array}{l}\text { Urinary tract infection, } \\
\text { veneral disease (Pradhan \& } \\
\text { Badola 2008); urinary tract } \\
\text { infection or inflammation } \\
\text { (Rai \& Sharma 1994) }\end{array}$ \\
\hline $\begin{array}{l}\text { Drymaria cordata } \\
\text { (L.) Willd. ex } \\
\text { Roem. \& Schult. }\end{array}$ & Whole plant & $\begin{array}{l}\text { Bone fracture, } \\
\text { mouth ulcer }\end{array}$ & Whole plant & $\begin{array}{l}\text { Sinusitis, nasal blockage, } \\
\text { headache, sore throat } \\
\text { pain, fever (Pradhan \& } \\
\text { Badola 2008); sinusitis } \\
\text { and nasal blockage } \\
\text { (Rai \& Sharma 1994) }\end{array}$ \\
\hline $\begin{array}{l}\text { Equisetum debile } \\
\text { Roxb. ex Vaucher }\end{array}$ & Stem shoots & $\begin{array}{l}\text { Stop bleeding after } \\
\text { injury, blood in urine } \\
\text { \& blood secretion } \\
\text { from vagina }\end{array}$ & Aerial parts & $\begin{array}{l}\text { Fresh wound, nose } \\
\text { bleeding, etc. to clot blood } \\
\text { (Pradhan \& Badola 2008) }\end{array}$ \\
\hline $\begin{array}{l}\text { Holarrhena } \\
\text { antidysenterica (L.) } \\
\text { Wall. ex A. DC. }\end{array}$ & Bark & $\begin{array}{l}\text { Constipation, } \\
\text { dysentery }\end{array}$ & $\begin{array}{l}\text { Bark, leaves, } \\
\text { latex }\end{array}$ & $\begin{array}{l}\text { Acute dysentery (Jana } \\
\text { \& Chauhan 2000); } \\
\text { amoebic dysentery (Rai } \\
\text { \& Sharma 1994) }\end{array}$ \\
\hline $\begin{array}{l}\text { Picrorhiza kurroa } \\
\text { Royle ex Benth. }\end{array}$ & Roots & $\begin{array}{l}\text { Cold and fever, } \\
\text { anorexia }\end{array}$ & Roots/rhizomes & $\begin{array}{l}\text { Cough, fever (Pradhan } \\
\text { \& Badola 2008); malarial } \\
\text { fever, catharitic, purgative } \\
\text { and dyspsiac (Rai \& } \\
\text { Sharma 1994) }\end{array}$ \\
\hline $\begin{array}{l}\text { Rhododendron } \\
\text { arboreum Sm. }\end{array}$ & Flowers & $\begin{array}{l}\text { Diarrhea and } \\
\text { dysentery }\end{array}$ & $\begin{array}{l}\text { Flowers, buds } \\
\text { and petals }\end{array}$ & $\begin{array}{l}\text { Blood dysentery (Dash } \\
\text { et al. 2003); dysentery } \\
\text { and diarrhea (Jana \& } \\
\text { Chauhan 2000) }\end{array}$ \\
\hline $\begin{array}{l}\text { Rhododendron } \\
\text { campanulatum } \\
\text { D. Don }\end{array}$ & Leaves & Chronic fever & Leaves & $\begin{array}{l}\text { Chronic rheumatism, } \\
\text { syphilis, sciatica (Jana \& } \\
\text { Chauhan 2000); cough } \\
\text { (Pradhan \& Badola 2008) }\end{array}$ \\
\hline Rubia cordifolia L. & Roots & $\begin{array}{l}\text { Skin infection, } \\
\text { postpartum recovery }\end{array}$ & Roots & $\begin{array}{l}\text { Urinary infection, skin } \\
\text { disease (Pradhan } \\
\text { \& Badola 2008) }\end{array}$ \\
\hline \multirow[t]{2}{*}{ Rubus ellipticus Sm. } & $\begin{array}{l}\text { New tender } \\
\text { leaves, young } \\
\text { stem shoots }\end{array}$ & Cold and fever & Young stem & Dysentery (Dash et al. 2003) \\
\hline & Roots & Wound & Dried roots & Jaundice (Dash et al. 2003) \\
\hline $\begin{array}{l}\text { Sinopodophyllum } \\
\text { hexandrum (Royle) } \\
\text { T.S. Ying }\end{array}$ & Roots/rhizomes & $\begin{array}{l}\text { Hoof infection, } \\
\text { indigestion }\end{array}$ & Roots & $\begin{array}{l}\text { Blood purifier, vermifuge, } \\
\text { purgative, emetic, } \\
\text { cholagogue, cardiac } \\
\text { tonic, skin inflammation } \\
\text { (Rai \& Sharma 1994) } \\
\end{array}$ \\
\hline $\begin{array}{l}\text { Swertia chirata } \\
\text { (Wall.) C.B. Clarke }\end{array}$ & Whole plant & Fever & Whole plant & Fever (Rai \& Sharma 1994) \\
\hline
\end{tabular}


Bharati \& Sharma - Plants used as Ethnoveterinary Medicines in Sikkim

Himalayas

\begin{tabular}{|l|l|l|l|l|}
\hline \multirow{2}{*}{ Species } & \multicolumn{2}{|l|}{ Veterinary medicine (Present study) } & Human medicine (Earlier studies) \\
\cline { 2 - 5 } & Part & Use & Part & Use \\
\hline $\begin{array}{l}\text { Terminalia belerica } \\
\text { (Gaertn.) Roxb. }\end{array}$ & Fruit & Diarrhea, dysentery & Fruit & $\begin{array}{l}\text { Indigestion, diarrhea } \\
\text { (Rai \& Sharma 1994) }\end{array}$ \\
\hline $\begin{array}{l}\text { Terminalia } \\
\text { chebula Retz. }\end{array}$ & Fruit/bark & Diarrhea, dysentery & Fruit & $\begin{array}{l}\text { Tonsillitis, pharyngitis and } \\
\text { other throat complications } \\
\text { (Rai \& Sharma 1994) }\end{array}$ \\
\hline \multirow{2}{*}{\begin{tabular}{l} 
Urtica dioica L. \\
\cline { 2 - 5 }
\end{tabular}} & Roots & Bone fracture & Roots & $\begin{array}{l}\text { Minor fracture (Rai } \\
\text { \& Sharma 1994) }\end{array}$ \\
\cline { 2 - 5 } & Young leaves & Galactagogue & Whole plant & $\begin{array}{l}\text { Bone fracture and } \\
\text { dislocation, diarrhea, } \\
\text { cough, child delivery } \\
\text { (Pradhan \& Badola 2008) }\end{array}$ \\
\hline $\begin{array}{l}\text { Zingiber officinale } \\
\text { Roscoe }\end{array}$ & Rhizomes & Cold and cough & Rhizomes & $\begin{array}{l}\text { Cough, fever, throat pain } \\
\text { (Pradhan \& Badola 2008) }\end{array}$ \\
\hline
\end{tabular}

Ethnoveterinary information on 37 plant species of Sikkim Himalayas was recorded. These plants are being used in 15 broad categories of livestock ailments: diarrhea and dysentery, digestive disorder, injury and wound, fever, maternity ailments, skin disease, urinary ailment, cough and cold, skeleto-muscular problems, inflammation, scorpion sting, snake and insect bite, weakness, worm, ulcer and bleeding.
Out of these 37 species, roots/rhizomes of 16 species were being harvested for 25 remedies, followed by leaves of 10 species for 13 remedies, whole plants of four species used to treat six remedies, fruits of three species for six remedies, stem \& stem bark of five species for five remedies, seed of three species for four remedies and flower of two species for two remedies (Figure 2). Herbs

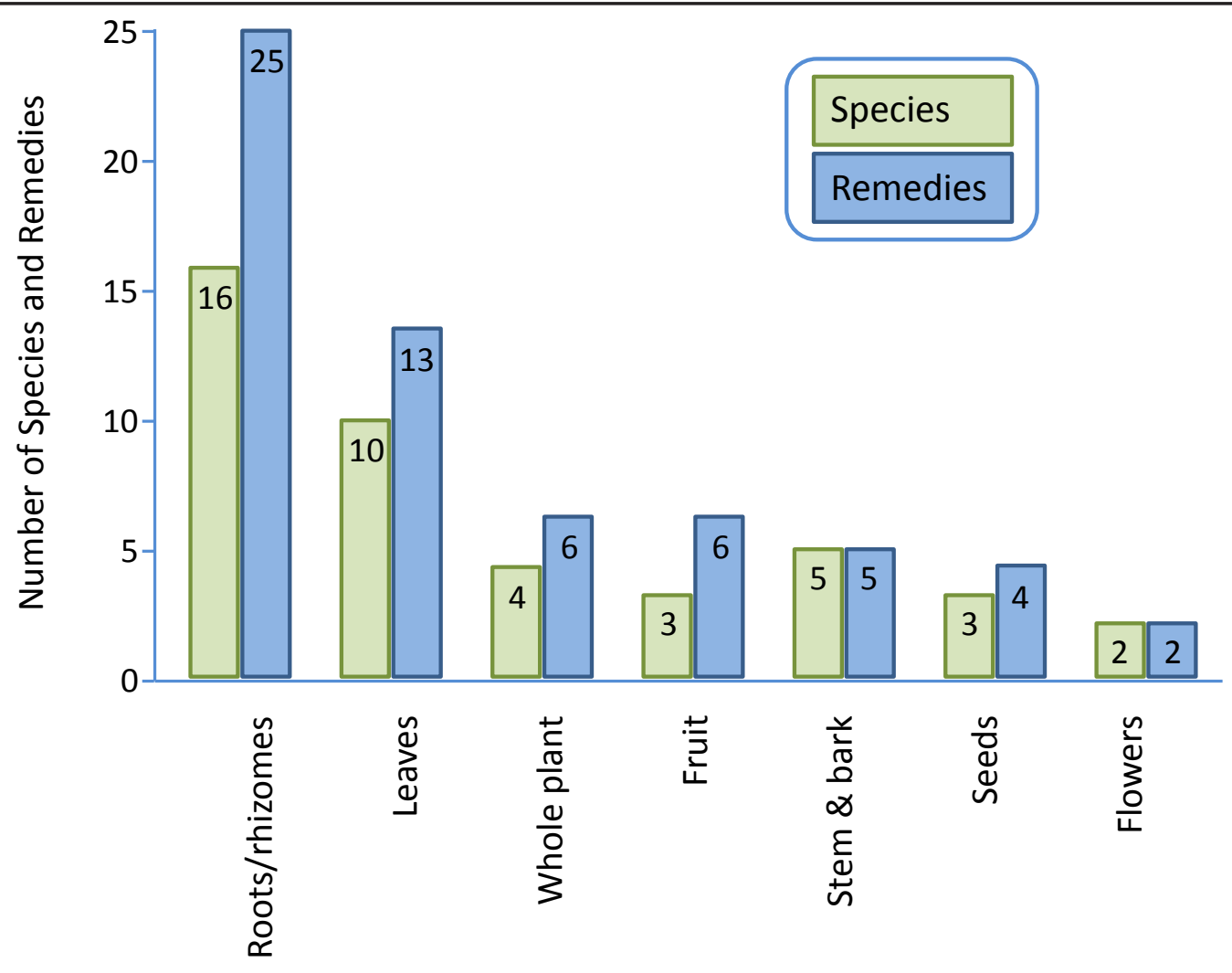

Plant Parts

Figure 2. Comparison of plant part(s) used between remedies and plant species, in ethnoveterinary medicine in Sikkim, Himilayas, India. 


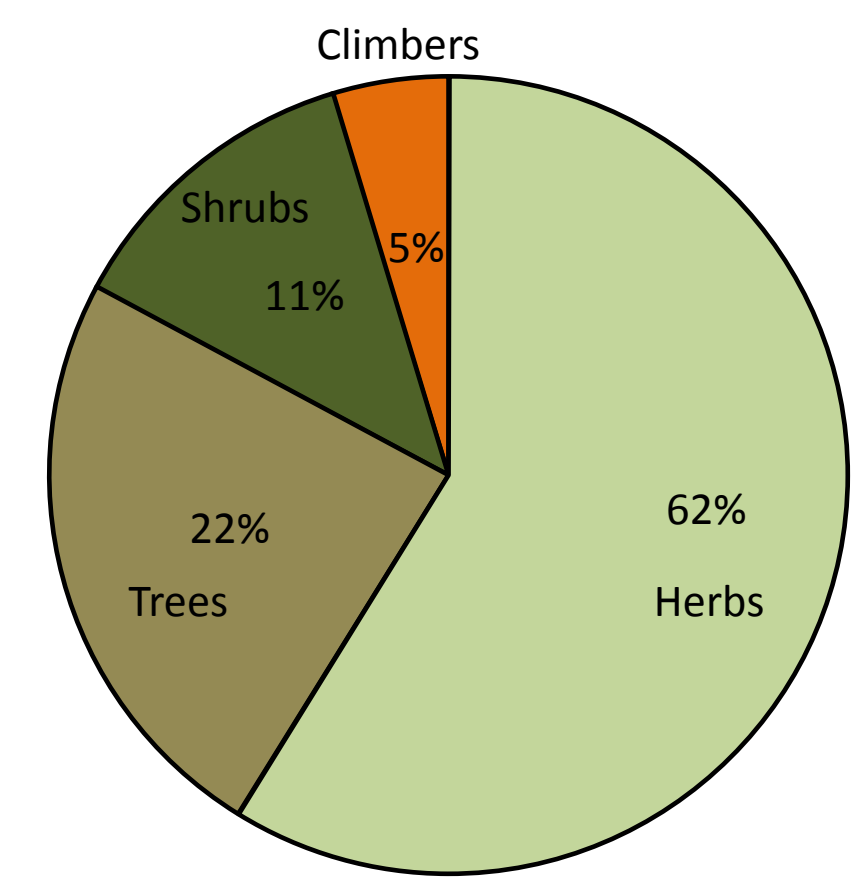

Figure 3. Medicinal plants used in ethnoveterinary medicine in Sikkim Himalayas, India arranged according to their life forms.

are the primary source of medicinal plants in terms of number of species $(62.16 \%$ ) followed by trees (Figure 3 ).

In the present study, nine species are used to treat diarrhea \& dysentery, followed by injury \& wounds by seven species, fever by seven species, digestive disorders by six species, maternity ailments by five species, skin disease by four species, urinary ailments by four species, cough \& cold by three species, skeleto-muscular problems by three species, inflammation by three species, scorpion sting, snake \& insect bite by three species, weakness by two species, worm by two species, ulcer by two species and bleeding by one species (Figure 2).

\section{Discussion}

\section{Comparative assessment}

This research recorded 13 species being used as ethnoveternary medicine in Sikkim as being used to treat similar ailments in other parts of India (Table 1). With 37 species being reported during this research, this indicates moderate affinity (37\%) between ethnoveterinary medicinal system of Sikkim and rest of the India. However, 12 species $(32.43 \%)$ have common medicinal use in both human and livestock. This suggests that the relationship between human and veterinary medicine has been parallel (Table 2).

\section{New findings}

Twelve plant 12 species $(32.43 \%$ of those recorded) were reported for the first time as being used as ethnoveternary medicine (Table 1). Further, this study reports new remedies which were not mentioned in previous studies. For example, participants reported use of Acorus calamus L. for indigestion and woundhealing, which is a use not previously documented. Similarly, use of Bauhinia variegata L. for diarrhea, use of Rhododendron campanulatum D. Don for fever, Cannabis sativa L. for inflammation, and use of Rauvolfia serpentina (L.) Benth. ex Kurz for snake-bite have not been reported in earlier studies on ethnoveternary medicines of the region (Table 1).

\section{Status of traditional knowledge}

It has been observed that older persons (age group 55-70 years) and traditional healers have greater knowledge about traditional medicines than younger persons (age group $30-40$ years). This may be due to increased awareness of allopathic treatment and modern medicines in younger people, however the traditional medicinal system is still prevalent in remote villages of Sikkim. The primary ailments of livestock are diarrhea, injury, fever, digestive disorders and maternity complications, which are commonly treated with medicinal plants.

Although hematuria is the most common livestock disease reported by the government veterinary hospital in Gangtok, villagers in the study area mentioned digestive disorders, such as anorexia, diarrhea, dysentery and indigestion as the most common ailments in Sik$\mathrm{kim}$. The probable reason behind such contradictions is use of home remedies to cure digestive disorders, hence they are not reported to veterinary hospitals.

\section{Conservation of species}

Several locally uncommon species, such as Bergenia ciliata Sternb. (Figure 5, Figure 6), Cissampelos pareira L. and Swertia chirata (Wall.) C. B. Clarke were reported as being destructively harvested for livestock medicines by using whole plant or roots/rhizomes, which may be related to their possible vulnerability of deplition. During the interview process, about $60 \%$ of participants mentioned $S$. chirata as being the most frequently used ethnoveternary species. This supports the the observations of Nayar and Sastry (1987-1990) that $S$. chirata is considered as vulnerable for northeast India. 


\section{Himalayas}

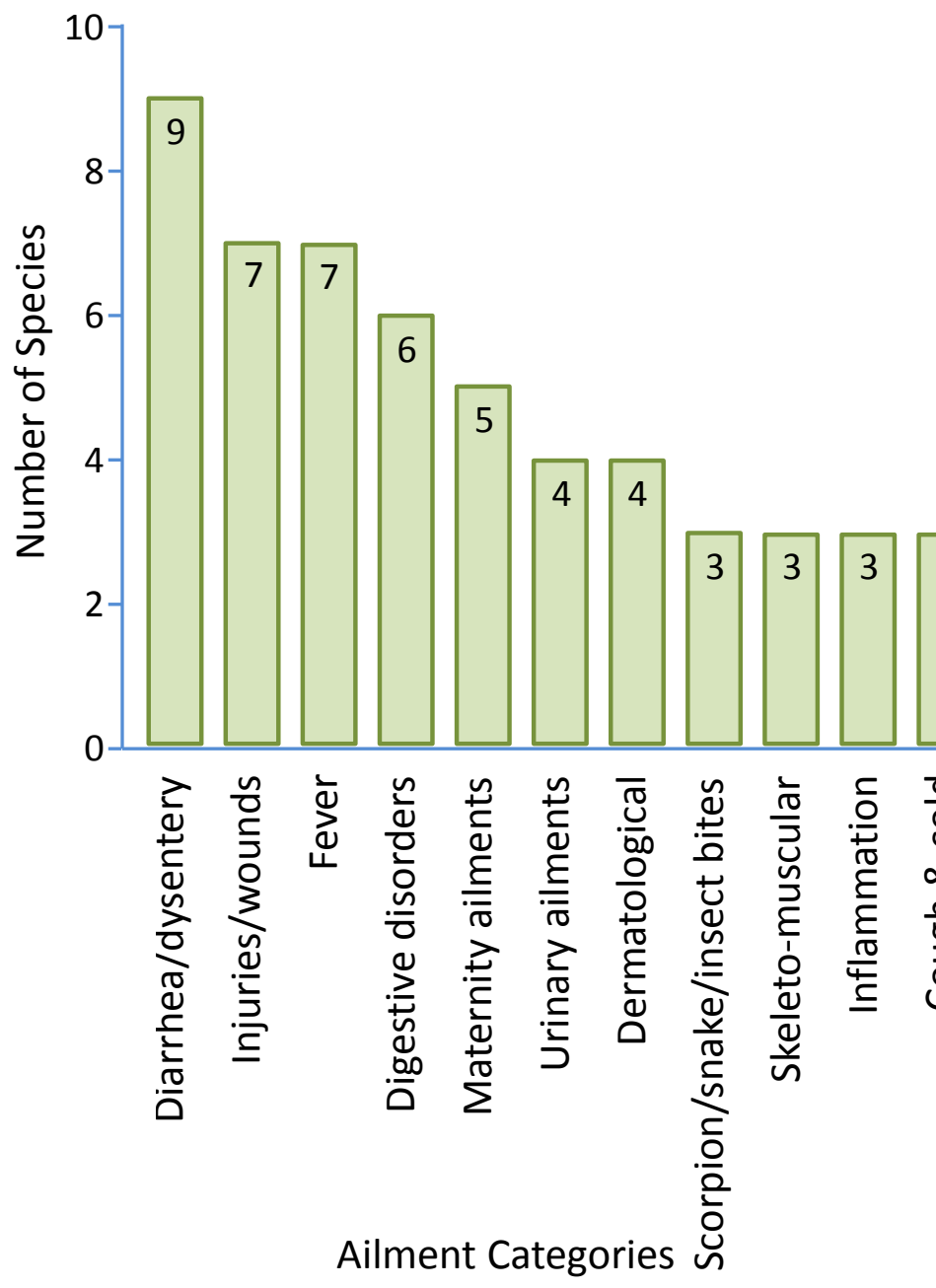

Figure 4. Major ailment categories and number of associated plant species used in ethnoveterinary medicine in Sikkim Himalayas, India.

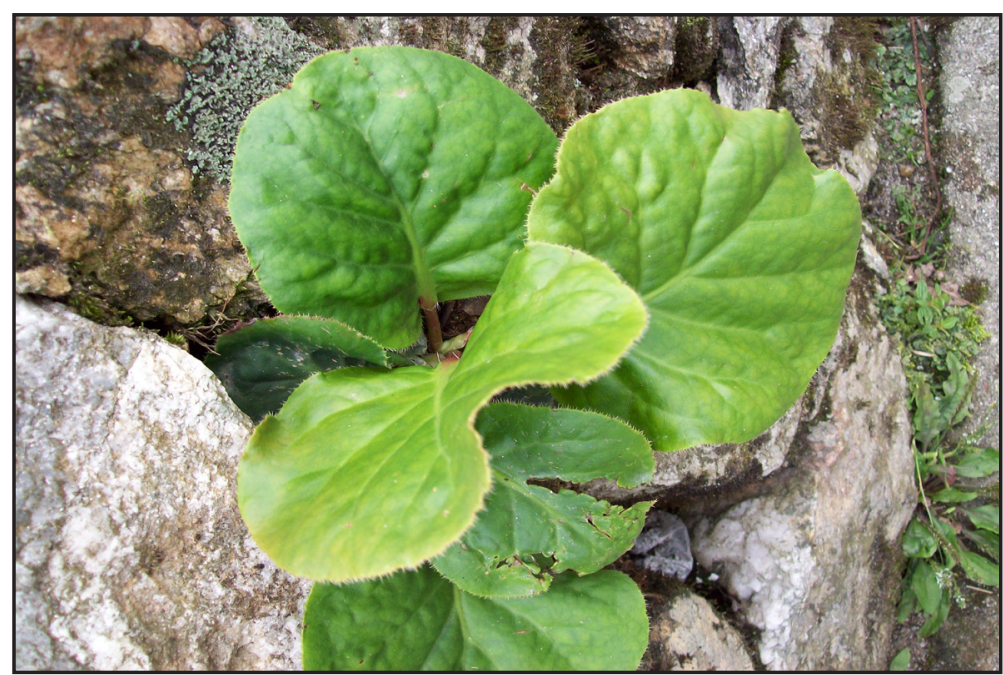

Figure 5. Bergenia ciliata Sternb. showing hairy and rounded leaves. $B$. ciliata is used in ethnoveterinary medicine in Sikkim Himalayas, India.

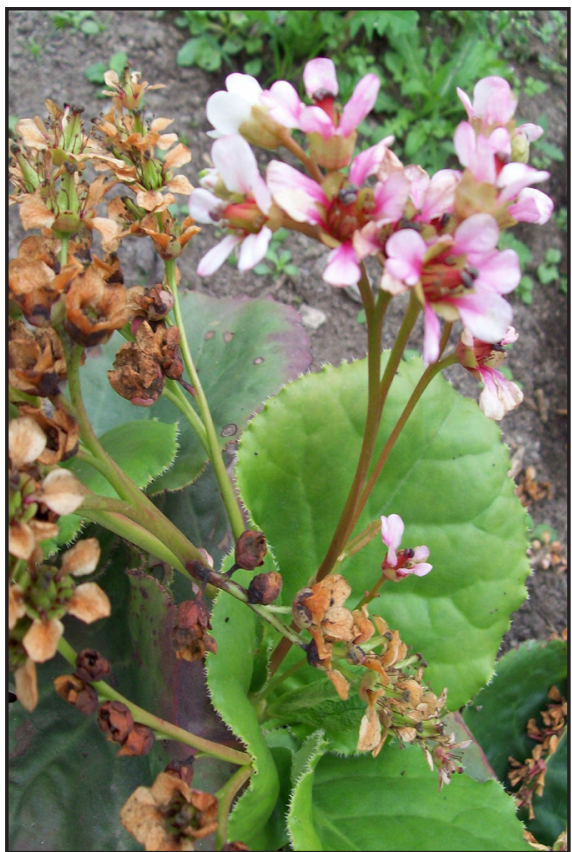

Figure 6. White-pink inflorescence of Bergenia ciliata Sternb. in Sikkim Himalayas, India. 
Other species, such as Acorus calamus L., Centella asiatica (L.) Urb. and Picrorhiza kurrooa Royle ex Benth. are also widely used medicinaly plants for humans and subjected to destructive harvesting through digging/uprooting the plant or roots/rhizomes. Hence, such medicinal plants need conservation protection in natural habitats, and large scale cultivation to take pressure off of these plants.

\section{Conclusion}

Traditional health care practices have been developed through generations of practical experience without external input. This study provides identifies ethnoveterinary plant species, potentially for economic development. Further study and promotion of ethnoveterinary medicine is bound to help the communities conserve information and integrate select practices into rural animal healthcare services. This may create opportunities for phytochemists and pharmacologists to conduct future studies. Two points are worth underlining: first, local knowledge can be converted into medicinal or other commercial products. Local people and the keepers of this knowledge should be recognized and appropriately compensated. Second, over-exploitation of medicinal plants is bound to put their survival at risk and measures need to be implemented to conserve them. The situation is made more urgent since in many regions both traditional knowledge and plant diversity are disappearing rapidly (Mathias 2007).

\section{Acknowledgements}

The authors would like to thank the scientists of FRI, Dehradun and BSI, Gangtok, for their valuable help with identification of plants and to Dr. H.B. Singh, Scientist, NISCAIR, New Delhi for his guidance rendered during preparation of this paper. Our special thanks to Julia M.S. Mudrick of Provo, Utah, United States for her valuable help with the language editing of this paper. Thanks are also due to the Council for Scientific and Industrial Research (CSIR), New Delhi, India for providing financial assistance in the form of a Senior Research Fellowship (SRF-NET).

\section{Literature Cited}

Anjaria, J., M. Parabia \& S. Dwivedi. 2002. Ethnovet Heritage: Indian ethnoveterinary medicine an overview. Pathik Enterprise, Ahmedabad, India.

Anonymous. 2006. Department of Animal Husbandry and Veterinary Services. Government of Sikkim, Gangtok, India.

Anonymous. 2008. Ministry of Health and Family welfare. Government of India, New Delhi.

Atkinson, E.T. 1882. The Himalayan Gazeteer. Volume Two. Reprint 1973. Cosmo Publication, Delhi, India.
Balaraman, N. \& M.M. Golay. 1991. Livestock Production in Sikkim. Sikkim Science Society, Gangtok, India.

Bandyopadhyay, S. \& S.Kr. Mukherjee. 2005. Ethnoveterinary medicine from Koch Bihar district, West Bengal. Indian Journal of Traditional Knowledge 4(4):456-461.

Bennet, S.S.R. 1983. Ethno-botanical Studies in Sikkim. Indian Forester 109(7):477-481.

Bennet, S.S.R. 1985. Ethnobotanical studies in West Sikkim. Journal of Economic and Taxonmic Botany 7:317321.

Biswas, K. 1956. Common Medicinal plants of Darjeeling and Sikkim Himalaya. Government of West Bengal, Commerce and Industries Department. Superintendent, Government Printing, West Bengal Government Press, Alipore, India.

Borthakur, S.K. \& U.K. Sarma. 1996. Ethnoveterinary herbal medicines in Morena District of Madhya Pradesh, India. Pp. 197-199 in Ethnobiology in Human Welfare. Edited by S.K. Jain. Deep Publications, New Delhi.

Burman, R.J.J. 2003. Tribal Medicine. Mittal Publications, New Delhi.

Chaudhary, K.C.R. 1951. Sikkim - the country and its forests. Indian Forester. 77:676-683.

Chopra, R.N., S.L. Nayar \& I.C. Chopra. 1956. Glossary of Indian Medicinal Plants. Council of Scientific and Industrial Research, New Delhi.

Clarke, C.B. 1876. Botanical notes from Darjeeling to Tonglo. Journal of the Linnean Society 15:116-159.

Cunningham, A.B. 2001. Applied Ethnobotany: People, wild plant use and conservation. Earthscan Publishing Limited, London.

Dash, S.S., A. Maiti \& S.K. Rai. 2003. Traditional uses of plants among the urban population of Gangtok - Sikkim. Pp. 313-324 in Ethnobotany and Medicinal Plants of India and Nepal. Volume One. Edited by V. Singh \& A.P. Jain. Scientific Publishers, Jodhpur, India.

Deshpande, D.J. 2006. A Handbook of Medicinal Herbs: A source book of herbal remedies. Agrobios, Jodhpur, India.

Dwivedi, S.K. 2003. Ethnoveterinary medicine in ancient India. Pp. 103-106 in Veterinary Science and Animal Husbandry in Ancient India. Edited by R. Somvanshi \& M.P. Yadav. IVRI, Izatnagar, India.

Gaur, R.D. 1992. An ethnobotanical study of Uttarpradesh in relation to veterinary medicines. Journal of the Indian botanical Society 72:139-144. 


\section{Bharati \& Sharma - Plants used as Ethnoveterinary Medicines in Sikkim Himalayas}

Ghotage, N.S. \& S.R. Ramdas. 2008. Plants Used in Animal Care: The Anthra collective. Anthra, Pune, India.

Hajra, P. K. \& P. Chakraborty. 1981. A survey of wild plants sold in the Lal market of Gangtok. Indian Journal of Forestry 4(3):217-220.

Hajra, P. K. \& D.M. Verma. 1996. Flora of Sikkim. Volume One. BSI, Kolkata, India.

Hara, H. 1966. The Flora of Eastern Himalaya. The University of Tokyo Press, Tokyo.

Harsha, V.H., V. Shripathi \& G.R. Hegde. 2005. Ethnoveterinary practices in Uttara Kannada district of Karnataka. Indian Journal of Traditional Knowledge 4(3):253-258.

Hooker, J. D. 1849. The Rhododendrons of Sikkim-Himalaya. Reeve, Benham and Reeve, London.

Jain, S.K. \& S. Srivastava. 1999. Dictionary of Ethnoveterinary Plants of India. Deep Publications. New Delhi.

Jain, S.K. \& S. Srivastava. 2003. Some folk herbal medicines for possible use in veterinary practices. Indian Journal of Traditional Knowledge 2(2):118-125.

Jain, S.K. 1981. Glimpses of Indian Ethnobotany. Oxford \& IBH Publishing Co. New Delhi.

Jana, S.K. \& A.S. Chauhan. 1999. Studies on Lepcha medico-botany of Dzongu, Sikkim Himalaya. Himalayan Paryavaran 6:121-126.

Jana, S.K. \& A.S. Chauhan. 2000. Ethnobiological studies on Lepchas of Dzongu, North Sikkim, India. Annals of Forestry 8(1):131-144.

King, G. \& R. Pantling. 1889. The orchids of the Sikkim Himalaya. Annals of Royal Botanic Garden Calcutta 8(24):1-448.

Kirtikar, K.R. \& B.D. Basu. 1935. Indian Medicinal Plants - with illustrations. Second edition. Volumes One - Four. Lalit Mohan Basu. Allahbad, India.

Kiruba, S., S. Jeeva \& S.S.M. Dhas. 2006. Enumeration of ethnoveterinary plants of Cape Comorin, Tamil Nadu. Indian Journal of Traditional Knowledge 5(4):576-578.

Krishna, B. \& S. Singh. 1987. Ethnobotanical observations in Sikkim. Journal of Economic and Taxonomic Botany $9(1): 1-7$.

Kumar, A.T. 2003. Veterinary Ayurveda in ancient Indian literature. Pp. 8-16 in Veterinary Science and Animal Husbandry in Ancient India. Edited by R. Somvanshi \& M. P. Yadav. IVRI, Izatnagar, India.
Maiti, D.C., A.S. Chauhan \& G. Maiti. 2003. Ethnonbotanical notes on some unexploited plants used by Lepchas and Nepalese communities of North Sikkim. Pp. 325-332 in Ethnobotany and Medicinal Plants of India and Nepal. Volume One. Edited by V. Singh \& A.P. Jain. Scientific Publishers, Jodhpur, India.

Mathias, E. 2007. Ethnoveterinary medicine in the era of evidence-based medicine: Mumbo-jumbo or a valuable resource? The Veterinary Journal 173:241-242.

Mehra, P.N. \& S.S. Bir .1964. Pteridophytic flora of Darjelling and Sikkim Himalayas. Resource Bulletin of Punjab University 15(1-2):69-181.

Mishra, S., V. Jha \& S. Jha. 1996. Ethno-veterinary herbal medicines in Morena district of Madhya Pradesh, India. Pp. 189-193 in Ethnobiology in Human Welfare. Edited by S.K. Jain. Deep Publications, New Delhi.

Myers, N., R.A. Mittermeier, C.G. Mittermeier, G.A. B. da Fonseca \& J. Kent. 2000. Biodiversity hotspots for conservation priorities. Nature. 403:853-858.

Nayar, M. P. \& A.R.K. Sastry. 1987- 1990. Red Data Book of Indian Plants. Volumes One - Three. Botanical Survey of India, Kolkata, India.

Pande, P.C., L. Tiwari \& H.C. Pande. 2007. Ethnoveterinary plants of Uttaranchal - A review. Indian Journal of Traditional Knowledge 6(3):444-458.

Pandey, H.P., B.K. Verma \& S. Narain. 1999. Ethnoveterinary plants of Gonda region. Journal of Economic and Taxonomic Botany 23(1):199-203.

Pradhan, B.K. \& H.K. Badola. 2008. Ethnomedicinal plant use by Lepcha tribe of Dzongu valley bordering Khangchendzonga Biosphere Reserve, in North Sikkim, India. Journal of Ethnobiology and Ethnomedicine 4:22.

Puri, H. S. \& G. Pandey. 1980. Glimpses in to the crude drugs of Sikkim. Bulletin of Medico-Ethno Botanical Research 1(1):55-71.

Rai, L.K. \& E. Sharma. 1994. Medicinal Plants of Sikkim Himalaya; Status uses and potential. G. B. Pant Institute of Himalayan Environment and Development, Gangtok, India.

Rao, S.R. 1963. Botanical tour in the Sikkim state. eastern Himalaya. Bulletin of the Botanical Survey of India 5(2):165-205.

Reddy, C.S. \& V.S. Raju. 2000. Folklore biomedicine for common veterinary diseases in Nalgonda district, Andhra Pradesh. Ethnobotany 12:113-117. 
Samal, P.K., A. Shah., S. Tiwari, B. Mehra \& D.K. Agrawal. 2003. Indigenous animal health care practices in Indian Central Himalaya: Emperical evidences. Indian Journal of Traditional Knowledge 2(1):40-50.

Shirlaw, L.H. 1940. A short history of Ayurvedic veterinary literature. Indian Journal of Veterinary Science 10:1-39.

Sikarwar, R.L.S. 1996. Ethno-veterinary herbal medicines in Morena district of Madhya Pradesh, India. Pp. 194-196 in Ethnobiology in Human Welfare. Edited by S.K. Jain. Deep Publications, New Delhi.

Singh, K.K. \& K. Kumar. 2000. Observations on ethnoveterinary medicine among the Gaddi Tribe of Kangra valley, Himachal Pradesh. Ethnobotany 12:42-44.

Singh, P. \& A.S. Chauhan. 1997. Plant diversity in Sikkim Himalaya. Pp. 137-162 in Plant Diversity Hotspots in India: An overview. Edited by P.K. Hajra \& V. Mudgal. Botanical Survey of India, Calcutta, India.

Smith, W.W. \& G.H. Cave. 1911. The Vegetation of Zemu and Llonakh Valley of Sikkim. Allied Book Centre, Dehradun, India.
Smith, W.W. 1913. The Alpine and Sub-alpine Vegetation of South-East Sikkim. Allied Book Centre, Dehradun, India.

Srivastava, G.N., S.A. Hasan, G.D. Bagchi \& S. Kumar. 2000. Indian Traditional Veterinary Medicinal Plants. ClMAP, Lucknow, India.

Swarup, D. 2003. Pashu Ayurveda during Vedic Period. P. 107 in Veterinary Science and Animal Husbandry in Ancient India. Edited by R. Somvanshi \& M.P. Yadav. IVRI, Izatnagar, India.

Tiwari, L. \& P.C. Pande. 2006. Indigenous veterinary practices of Darma valley of Pithoragarh district, Uttranchal. Indian Journal of Traditional Knowledge 5(2):201-206.

Tiwari, L. \& P.C. Pande. 2004. Traditional veterinary practices in south-eastern part of Chamoli district, Uttaranchal. Indian Journal of Traditional Knowledge 3(4):397-406. 